\title{
Acknowledgement to Reviewers of Applied Sciences
} in 2016

\author{
Applied Sciences Editorial Office,
}

Published: 12 January 2017

MDPI AG, St. Alban-Anlage 66, 4052 Basel, Switzerland; applsci@mdpi.com

The editors of Applied Sciences would like to express their sincere gratitude to the following reviewers for assessing manuscripts in 2016.

We greatly appreciate the contribution of expert reviewers, which is crucial to the journal's editorial process. We aim to recognize reviewer contributions through several mechanisms, of which the annual publication of reviewer names is one. Reviewers receive a voucher entitling them to a discount on their next MDPI publication and can download a certificate of recognition directly from our submission system. Additionally, reviewers can sign up to the service Publons (https://publons.com) to receive recognition. Of course, in these initiatives we are careful not to compromise reviewer confidentiality. Many reviewers see their work as a voluntary and often unseen part of their role as researchers. We are grateful to the time reviewers donate to our journals and the contribution they make.

If you are interested in becoming a reviewer for Applied Sciences, see the link at the bottom of the webpage http://www.mdpi.com/reviewers.

The following reviewed for Applied Sciences in 2016:

Abbod, Maysam F.
Abdelaziz, Hamzaoui
Abdelkarim, Omar I.
Abdelkefi, Abdessattar
Abdelsalam, Ibrahim
Abdollahzadeh Jamalabadi, M. Y.
Abdulshahed, Ali
Abraham, John P.
Acharya, U. Rajendra
Adams, Matthew P.
Adams, Samantha V.
Adeeb, Samer
Afreixo, Vera
Aggelis, Dimitrios
Aggogeri, Francesco
Agrela, Francisco
Agresti, Filippo
Aguado, Sergio
Ahmed, Ehab
Ahmed, Hafiz

Ahn, Changbum
Ahrens, Jens
Aillerie, Michel
Akhavan, Omid
Akhlaghi, Shahin
Alagan Chella, Mayilvahanan
Alam, Morshed
Alamaniotis, Miltiadis
Alander, Jarmo
Alavi, Amir H.
Albo, Jonathan
Aleixos, Nuria
Aletta, Francesco
Alexandre, Gladys
Ali, Mohd Hasan
Alidaee, Bahram
Alleaume, Samuel
Alles, Erwin Jozef
Almeida, Tiago Paggi
Almond, Darryl P.

\author{
Altarawneh, Mohammednoor \\ Amankwah, Emmanuel K. \\ Amano, Ken-ichi \\ Ambrosi, Adriano \\ Ameli, Amir \\ Amezcua, Javier \\ Amir, Fatima Z. \\ Amorati, Riccardo \\ Anderson, Anne J. \\ Angelini, Angelo \\ Angiolini, Federico \\ Anguita, Davide \\ Annamdas, Venu G. M. \\ Anne, Jozef \\ Annema, Anne-Johan \\ Antolini, Francesco \\ Antonopoulos, Angelos \\ Appetecchi, Gianni B. \\ Arahal, Manuel R. \\ Arbabi, Vahid
}


Arena, Andrea

Argyropoulos, Christos

Ariga, Katsuhiko

Armon, Robert

Arnaut, Luk

Arora, Ashish

Arshad, Muhammad

Artal, Sergio

Arvapally, Ravi Kumar

Aryan, Pouria

Asadi, Ehsan

Asami, Toshihiko

Asamizu, Shumpei

Asdrubali, Francesco

Astolfi, Arianna

Astolfi, Jacques-André

Atamturktur, Sez

Athanasiou, Lampros

$\mathrm{Au}$, Man Ho

Aumond, Pierre

Avila-Sanchez, Sergio

Aw, Tiong Gim

Axelsson, Östen

Aymerich, Francesco

Azman, Samet

Azmi, Azwan Iskandar

Babu, S. V.

Bae, Euiwon

Bae, Yoon Yeong

Baer, Thomas

Bagci, Ulas

Bahadur, Vaibhav

Baker, Kenneth R.

Baker, Thar

Baldi, Simone

Ban, Tae-Won

Banach, Marcin

Banerjee, Sean

Bansil, Rama

Bao, Jie

Baranov, Denis

Barbancho, Ana M.

Barbancho, Isabel
Barbillon, Grégory

Barbosa, Ramiro S.

Bard, Delphine

Barea, Eva M.

Barron, Andrew R.

Barros, Antonio

Barshtein, Gregory

Barucca, Gianni

Basner, Mathias

Bayer, Ilker S.

Beauchamp, James

Bechhoefer, Eric

Bednarski, Patrick

Bedon, Chiara

Begum, Shamim A.

Behnood, Ali

Bell, Carl-Martin

Bellido, Francisco J.

Bencivenga, Filippo

Ben-Idris, Mohammed

Benim, Ali Cemal

Benini, Ernesto

Berardi, Umberto

Berendsen, Floris F.

Bergada, Josep Maria

Bernacki, Bruce

Berretti, Stefano

Berselli, Giovanni

Berto, Filippo

Berzina, Tatiana

Beskhyroun, Sherif

Besser, Charlotte

Besson, Adrien

Bevan, Kirk H.

Beyerer, Jürgen

Bhinder, Majid A

Bianchi, Francesca Antonella

Bianco, Vincenzo

Biderman, Michael

Birch, John

Birken, Philipp

Biswas, Roshni

Bitting, Rhonda L.
Björling, Marcus

Blaabjerg, Frede

Blázquez, Javier S.

Blesa, Joaquim

Boada, Beatriz L.

Boege, Fritz

Boets, Pieter

Boisseau, Sebastien

Boland, Frank

Boldor, Dorin

Bolognani, Silverio

Bonacina, Luigi

Bonali, Fabio Luca

Bönninger, Ingrid

Booth, Brian

Borchers, Christine

Borderon, Caroline

Borghetti, Brett

Borgne, Yann-Ael Le

Borla, Oscar

Börner, Anko

Borrego-Varillas, Rocío

Bose, Chris

Bossis, Georges

Botsis, John

Botteron, Cyril

Bottomley, Paul A.

Bouma, Henri

Bouras, Christos

Bouzid, Allal M.

Boys, John T.

Bradley, Justin M.

Braeken, An

Brambilla, Giovanni

Brand, Uwe

Bräuer-Burchardt, Christian

Brekken, Reidar

Brischetto, Salvatore

Brischke, Christian

Broekhuis, Antonius A.

Brójo, Francisco

Brunner, Andreas J.

Brunskog, Jonas 


\author{
Brutti, Alessio \\ Bucchi, Francesco \\ Bukkapatnam, Satish T.S. \\ Bunge, Christian-alexander \\ Burgio, Allessandro \\ Burkart, Ralph \\ Buset, Jonathan M. \\ Bustillo, Andres \\ Butcher, Thomas \\ Caballero, Emilio Rodríguez \\ Cabboi, Alessandro \\ Cabeza, Rafael \\ Cabral, João M. G. \\ Cabrales, Luis \\ Caccavale, Fabrizio \\ Cacciato, Giuseppe \\ Caetano, Marcelo \\ Cairns, Warren Raymond Lee \\ Calado, João M. F. \\ Calderón, Verónica \\ Camacho López, Ana María \\ Camacho, Antonio \\ Cambiaso, Enrico \\ Camões, Aires \\ Campos, Armando \\ Campuzano Ruiz, Susana \\ Can, Arnaud \\ Cancelli, Andrea \\ Cancelliere, Piergiacomo \\ Cannas, Marco \\ Cannone Falchetto, Augusto \\ Canteli, Alfonso Fernández \\ Cao, Pan \\ Capsoni, Antonio \\ Carabin, Giovanni \\ Carbonari, Luca \\ Cardile, Giuseppe \\ Carette, Jérôme \\ Carnevale, Mauro \\ Carrera, Erasmo \\ Carrillo, Alfonso Pérez \\ Carroll, Lee \\ Casalino, Giuseppe
}

Casals-Terré, Jasmina

Caschera, Daniela

Casciati, Sara

Casnati, Alessandro

Castellani, Francesco

Castellazzi, Giovanni

Castelli-Dezza, Francesco

Castiglioni, Carlo Andrea

Catalano, Pasquale

Cavagnino, Davide

Cavallini, Massimiliano

Caviglione, Luca

Ceola, Serena

Chadli, Mohammed

Chai, Rifai

Chalioris, Constantin E.

Chan, Kwok Sum

Chan, Nei-Li

Chan, Woei-Leong

Chan, Yung-Kuan

Chandrahalim, Hengky

Chang, En-Chih

Chang, Hsueh-Hsien

Chang, Jeang-Lin

Chang, Kang-Ming

Chao, Yu-Chiang

Charmet, Andrea Pietropolli

Charrier, Guillaume

Chatzi, Eleni

Cheer, Jordan

Chen, Boyang

Chen, Chieh-Li

Chen, Chiung Yao

Chen, Der-chin

Chen, Gang

Chen, Hong

Chen, Hui-Chi

Chen, Jenq-Shyong

Chen, Jian-Zhang

Chen, Jih-Jung

Chen, Jing-heng

Chen, Liangbiao

Chen, Shih-Hung
Chen, Shiuan-Yeh

Chen, Wenyu

Chen, Xuemin

Chen, Yi-Chung

Chen, Yu-Chang

Chen, Zhaoyu

Cheng, Bo

Cheng, Chin-hsing

Cheng, Kuan-Chen

Cheng, Xingguo

Chesneau, Xavier

Cheung, Ada S.

Cheung, Karen

Chew, Kok-Hon

Chiaravalloti, Agostino

Chiementin, Xavier

Chih, Mingchang

Chikashi, Nakamura

Chin, Cheng Siong

Chin, Wee Shong

Chirarattananon, Pakpong

Chiu, Chien-Kuo

Chiu, Justin N.W.

Cho, Byoung-kwan

Cho, Chang-Hee

Cho, Dong-Woo

Cho, Kyuman

Cho, Young-Ho

Choi, Baehoon

Choi, Hyo-Jick

Choi, Ji-Woong

Choi, Nag Jung

Choi, Seung-Bok

Chong, Alvin

Choudhury, Deepak

Choung, Joonmo

Christofa, Eleni

Christofides, Panagiotis D.

Christov, Nicolai

Chromik, Richard R.

Chryssolouris, George

Chu, Dafeng

Chu, Shao-I 


\begin{tabular}{|c|}
\hline Chuang, Ta-hsien \\
\hline Chung, Kung-Ming \\
\hline Chung, Myong-Soo \\
\hline Chung, Tai-Shung \\
\hline Chung, Yi-Shih \\
\hline Chung-Chi, Huang \\
\hline Ciaccheri, Leonardo \\
\hline Cimato, Stelvio \\
\hline Cinca, Núria \\
\hline Cintuglu, Mehmet Hazar \\
\hline Claverley, James D. \\
\hline Clochard, Marie-Claude \\
\hline Cocolios, Thomas Elias \\
\hline Coelho, Luis \\
\hline Colwell, John M. \\
\hline Combroux, Isabelle \\
\hline Comyn-Wattiau, Isabelle \\
\hline Concilio, Antonio \\
\hline Conejero, Alberto \\
\hline Congedo, Paolo Maria \\
\hline Corkery, Gerard \\
\hline Coronas, Joaquín \\
\hline Corradi, Marco \\
\hline Cortazar, Osvaldo Daniel \\
\hline Cortell, Jason \\
\hline Corujo, Daniel \\
\hline Costanzo, Sandra \\
\hline Costa-Requena, Jose \\
\hline Coto, Baudilio \\
\hline Cox, Andrew M. \\
\hline Cozzolino, Daniel \\
\hline Crisostomi, Emanuele \\
\hline Croccolo, Dario \\
\hline Crussiere, Matthieu \\
\hline Cuce, Erdem \\
\hline Cucini, Riccardo \\
\hline Cuesta González, Eduardo \\
\hline Cunha, Angela \\
\hline Cupelli, Marco \\
\hline Curado, Marilia \\
\hline Custídio, João \\
\hline D'Alessandro, Walter \\
\hline Da Ros, Francesco \\
\hline
\end{tabular}

Daghooghi, Mohsen

Dai, Gaoliang

Dal Santo, Vladimiro

Dalton, Larry

Daly, Ian

D'Amato, Francesco

Dammak, Lasâad

Da'na, Enshirah

Danaila, Luminita

Daniele, Passeri

D'Aniello, Mario

Danno, Atsushi

Dantus, Marcos

Das, Amit

Das, Manash R.

Davidson, James S.

Davis, Elizabeth

Davis, Kristopher

De Aza, Piedad N.

De Brito, Jorge

De la Sen, Manuel

De Lacalle, L. N. López

De Marchi, Luca

De Marco, Iolanda

De Masi, Rosa Francesca

De Sousa, Ricardo J. Alves

De Vicente, Juan

De Zea Bermudez, Verónica

De Zordi, Nicola

Dehzangi, Abdollah (Iman)

Del Pino, Pablo

Del Pizzo, Silvio

Del Valle, Luis J.

Delgado, Juan Luis

Delis, Alex

Della-Morte, David

Dellepiane, Silvana

Demas, Nicholaos

Demers, Vincent

Demi, Libertario

Denbaars, Steven P.

Deng, Peng

Deng, Yaohua
Deo, Randhir

Dertimanis, Vasilis K.

Desai, Alok

Di Luzio, Giovanni

Diamantis, Konstantinos

Dias, Juliana

Diaz, Nelson L.

Dickert, Franz

Diego, Luna Martínez

Dimitrievska, Mirjana

Ding, Fei

Ding, Ing-Jr

Diraco, Giovanni

Diroll, Benjamin

Divall, Marta

Divekar, Prasad

Dixon, Simon

Dizqah, Arash M.

Djaoued, Yahia

Domaille, Dylan

Domaneschi, Marco

Donaire-Avila, Jesús

Donohue, John M.

Dordizadeh, Peyman

Dos Santos, Filipe Amarante

Dowling, Walter Jay

Drimus, Alin

Drissi-Habti, Monssef

Du, Hongjian

Duann, Jeng-Ren

Duchaine, Florent

Ducobu, François

Duma, Virgil Florin

Dunnigan, Matt

Duong, Minh Quan

Dupuis, Raphaël

Dworkin, Seth

Ebara, Mitsuhiro

Edirisinghe, Mohan

Edwards, Rodger

Egels, Matthieu

Eisemann, Martin

Eisenbarth, Hedwig 


Ekonomou, Lambros
Elbert, Philipp
El-Fiky, Eslam
Elgendi, Mohamed
Ellahi, Rahmat
Elleuche, Skander
El-Safty, Sherif A.

Emmerling, Franziska

Endo, Tatsuro

Erb, Uwe

Erdem, Emre

Erro, Daniel

Escuredo, Olga

Esfandyarpour, Rahim

Esmaeili, Mohammad

Ewels, Chris

Ewoldt, Randy

Fabrizio, Enrico

Fabrizio, Quadrini

Facchini, Francesco

Falcone, Francisco

Falconer, Robert J.

Fang, Peng

Fang, Shih-Hau

Fang, Steven

Fantuzzi, Nicholas

Faraldos, Marisol

Faravelli, Lucia

Farhangi, Hassan

Faruqi, Mohamed A.

Favier, Julien

Fazzolari, Fiorenzo

Fearn, Tom

Feng, Dongming

Fernandes, Emanuel M.

Fernandes-Ferreira, Manuel

Fernandez, Ines

Fernández-Blanco, Enrique

Fernandez-Llatas, Carlos

Ferracuti, Francesco

Ferrario, Valerio

Ferreira, Angela

Ferreira, José Vasconcelos
Feuer, Arie

Ficarella, Antonio

Fiebig, André

Fiebig, Uwe-Carsten

Figueiras-Vidal, Aníbal R.

Fiore, Vincenzo

Fiorino, Luigi

Fischer, Holger

Fischer, Peer

Fister, Iztok

Flanagan, Sara V.

Fleury, Martin

Flynn, Brian

Focacci, Francesco

Foglia, Gianmaria

Foglietta, Chiara

Fonseca, Ana

Fontana, Federico

Fontanesi, Stefano

Fortuna, Luigi

Foster, Shanelle

Fotouhi, Abbas

Fowler, David

Fox, Glenn

Fox, Nathan;

Franke, Jörg

Frano, Rosa Lo

Freeman, Chris

Freitas, Filomena

Frey, Georg

Frisenda, Riccardo

Frontoni, Emanuele

$\mathrm{Fu}$, Xingang

Fuentes, Raul

Fujita, Hamido

Fujita, Takayuki

Fung, Rong-fong

Furukawa, Ryo

Furutani, Katsushi

Furutani, Ryoshu

Gabriel, Jose Luis

Gabrielli, Leonardo

Gagnon, Joel
Galbrun, Laurent

Gallais, Laurent

Gallego, Antolino

Galván-D'Alessandro, Leandro

Gan, Tat-Hean

Gannon, Anthony J

Gao, Xiaohong

Gaponenko, Maxim

Garcia, Maria Da Luz

Garcia-Mina, Jose Maria

Gardeniers, Han

Garg, Akhil

Garrido, Piedad

Gasbarri, Paolo

Gaudino, Roberto

Gavaises, Manolis

Gazzola, Enrico

Geem, Zong Woo

Gensch, Michael

Gente, Ralf

Gentile, Piergiorgio

Georgoulas, George

Gerke, Jörg

Ghanbarian, Behzad

Ghimire, Deepak

Ghosh, Ambarnil

Giakoumidis, Ilias

Giaouris, Damian

Gibson, Christopher T.

Gil Del Val, Alain

Gil, Maria Luisa Almoraima

Gilmour, Jim

Giordano, Bruno

Giraud, Frédéric

Gismondi, Angelo

Gitz, Dennis C.

Gjestland, Truls

Godin, Nathalie

Goicoechea, Nieves

Gökce, Bilal

Goltsov, Alexandr

Goltz, Douglas M.

Gomes, Pedro 


Gomez Chavez, Karina Mabell
Gómez, Emilia
Gómez-Hernández, Jesús
Gonçalves, Rodrigo
Gonzalez Zalba, Miguel Fernando
González, Francisco
Gonzalez, Itziar
González, María de las Nieves
Gonzalez-Escribano, Arturo
González-Fonteboa, Belén
Gonzalez-Pedro, Victoria
González-Vázquez, Jesús
Gope, Prosanta
Goudarzi, Navid
Goula, Athanasia M.
Goyal, Raka
Graciani, Enrique
Graditi, Giorgio
Graff, Claus
Graffi, Kalman

Graumann, Peter L.

Gravenkamp, Hauke

Grazia De Giorgi, Maria

Grecco, Luca

Grenfell, James

Grimmelsman, Kirk

Grinblat, Gustavo

Grizeau, Dominique

Gross, Andreas

Gross, George

$\mathrm{Gu}$, Fengshou

Guan, Dongsheng

Guan, Yan

Guastavino, Catherine

Gude, Veera Gnaneswar

Gudi, Nikhil

Guerrero, Juan I.

Guihéneuf, Freddy

Günther, Matthias

Guo, Youguang

Gutiérrez Peña, Pedro Antonio

Gutierrez, Diego

Guzman, Marcelo
Gwinn, Kimberly

Ha, Yeong-Ho

Haddag, Badis

Haffner, Christian

Hagiwara, Yuki

Hak, Yi

Hall, Neal

Halpegamage, Sandamali

Hamada, Yuki

Han, Dong Wook

Han, Hao

Han, Qing-Long

Han, Seog-young

Han, Soonhung

Han, Yilong

Hann, Christopher

Hanoun, Samer

Hanson, Ronald K.

Hanusa, Timothy

Haq, Rizwan

Harle, Robert

Harnefors, Lennart

Haroldo, Maestre

Hase, Alan

Hasegawa, Satoshi

Hashemian, Reza

Haurie, Laia

Hausotte, Tino

Hautiere, Nicolas

Hayajneh, Khaled F.

Heap, Michael J.

Heckel, Frank

Heffernan, Jane M.

Heidari, Alireza

Helfield, Brandon L.

Hellinckx, Peter

Helsen, Lieve

Hennebelle, François

Henning, Andrew

Her, Shiuh-Chuan

Hernández, José G.

Hernández-García, Carlos

Heuberger, Manfred
Heutschi, Kurt

Hidema, Ruri

Hieu, Tran Dinh

Hilton, James E.

Hinderliter, Brian

Hirose, Yuichi

Ho, Johnny

Hofmann, Martin

Holzinger, Andreas

Hong, Keum-Shik

Hong, Sung-Nam

Hong, Ying-Yi

Hopkins, Simon C.

Horner, Andrew

Hosier, Ian L

Hosório, Hugo

Hossain, Mahtab

Hossain, Mohammad Billal

Hou, Shuhn-Shyurng

Howell, Bob A.

Hsiao, Fu-yuen

Hsiao, Kai-Long

Hsiao, Yu-Jen

$\mathrm{Hu}$, Jiefeng

$\mathrm{Hu}$, Jong Wan

$\mathrm{Hu}$, Weihao

$\mathrm{Hu}$, Weiping

Huang, Chih-Ching

Huang, Chih-Chung

Huang, Fuzhi

Huang, Jinxin

Huang, Ke

Huang, Po-Chin

Huang, Shyh-Chour

Huang, Xiao Liang

Huang, Xiaodi

Huang, Yu-hsi

Hueso, Jose L.

Huete, Juan F.

Hunstig, Matthias

Huston, Dryver

Hutmacher, Dietmar W.

Huynh, Nam Trung 


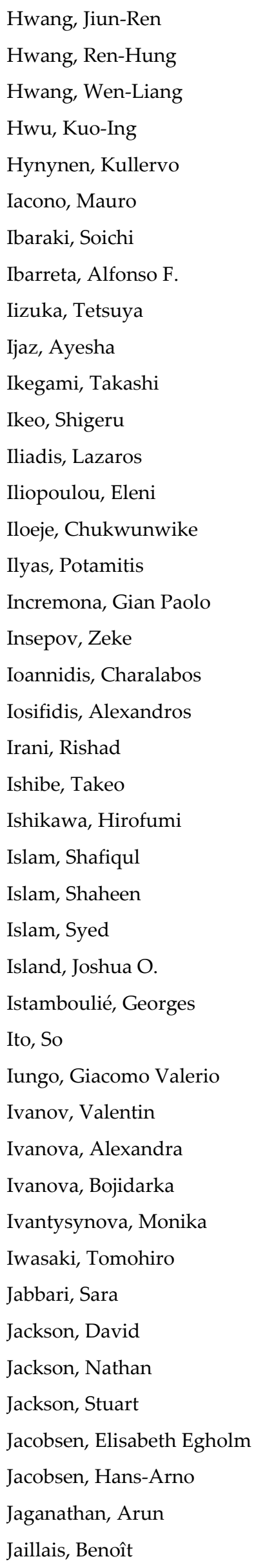

\begin{tabular}{|c|c|}
\hline Jalal, Ahmad & Kan, Chi-wai \\
\hline Jang, Choon-Man & Kanarachos, Stratis \\
\hline Jang, Soohwan & Kang, Dae-Ki \\
\hline Jansen, J. W. & Kang, Hyun Wook \\
\hline Jantunen, Erkki & Kang, Sanggyu \\
\hline Jariwala, Deep & Kanie, Kiyoshi \\
\hline Jatana, Gurneesh S. & Kantola, Raimo \\
\hline Jedermann, Reiner & Karaiskos, Grigorios \\
\hline Jehel, Pierre & Karbaschian, Mohammad Ali \\
\hline Jeng, Yih & Karimi, Hamid Reza \\
\hline Jensen, Annette Nygaard & Karlsson, Amy J. \\
\hline Jeon, Han-Yong & Karlsson, Stefan \\
\hline Jeon, Jin Yong & Karthikeyan, P. \\
\hline Jeon, Kwang Myung & Kartsakli, Elli \\
\hline Jhe, Wonho & Kašalynas, Irmantas \\
\hline Jia, Yu & Kashino, Kunio \\
\hline Jiang, Liben & Kasneci, Enkelejda \\
\hline Jiang, Ruinian & Kateris, Dimitrios \\
\hline Jiang, Xi & Kauhaniemi, Kimmo \\
\hline Jiang, Yifei & Kauko, Leiviskä \\
\hline Jiang, Zhengyi & Kauppinen, Anu \\
\hline Jimenez, Felipe & Kauranne, Tuomo \\
\hline Jiménez, José A. & Kavalenka, Maryna N. \\
\hline Jin, Maolin & Kawakita, Hidetaka \\
\hline Jin, Rize & Kellett, Christopher \\
\hline Jing, Xingjian & Kengne, Jacques \\
\hline Johnson, Steven L. & Kennel, Ralph \\
\hline Joshi, Simon & Ker, Jun-ing \\
\hline Joshi, Suraj & Keresztes, Janos \\
\hline Jost, Thomas & Kessler, Sylvia \\
\hline Jot, Jean-Marc & Khalaf, Abedawn I. \\
\hline Jotshi, Chand & Khalaf, Walaa \\
\hline Julian, Tim & Kharkivskiy, Sergiy \\
\hline Jun, Jong-gab & Kharrat, Mohamed \\
\hline Jung, Andras & Khazdozian, Helena \\
\hline Jung, Yeonwoong & Khennane, Amar \\
\hline Kacem, Najib & Khlissa, Radhouane \\
\hline Kaddoum, Georges & Khodadadi, Jay M. \\
\hline Kahle, Eckhard & Khodadoust, Amid P. \\
\hline Kakimoto, Koichi & Khoshmanesh, Khashayar \\
\hline Venkiteswaran, V. K. & Kim, Cheolhee \\
\hline Kamaly, Nazila & Kim, Cheong Ghil \\
\hline Kamphuis, I. G. & Kim, Chulhong \\
\hline
\end{tabular}




\begin{tabular}{|c|}
\hline Kim, Dai-Sik \\
\hline Kim, Dongwon \\
\hline Kim, Han-do \\
\hline Kim, Hyun Jae \\
\hline Kim, Hyun-Seok \\
\hline Kim, Hyunsung \\
\hline Kim, Jae-Kon \\
\hline Kim, Jeongyong \\
\hline Kim, Ji-Hee \\
\hline Kim, Jung Ho \\
\hline Kim, Jung Tae \\
\hline Kim, Kihyun \\
\hline Kim, Kikang \\
\hline Kim, Kwang Soo \\
\hline Kim, Kyo-Seon \\
\hline Kim, Myung Jun \\
\hline Kim, Sang Jae \\
\hline Kim, Sumin \\
\hline Kim, Sung-Kwon \\
\hline Kim, Sungwon \\
\hline Kim, Yong-Kab \\
\hline Kim, Young Dok \\
\hline Kinder, David \\
\hline King, Jerry W \\
\hline Kinnell, Peter \\
\hline Kirke, Alexis \\
\hline Kirtley, James L. \\
\hline Kitayama, Fumiya \\
\hline Knizley, Alta \\
\hline Ko, Jeong Hoon \\
\hline Ko, Seung Hwan \\
\hline Koduru, Janardhan Reddy \\
\hline Koehl, Patrice \\
\hline Kofoed, Jens Peter \\
\hline Kohlmann, Holger \\
\hline Kohno, Masamichi \\
\hline Kolb, Andreas \\
\hline Kolomenskiy, Dmitry \\
\hline Konstantinou, Georgios \\
\hline Koo, Bon \\
\hline Kormányos, Andor \\
\hline Korn, Tobias \\
\hline Korobenko, Artem \\
\hline
\end{tabular}

Kosasih, Buyung

Kostoglou, Margaritis

Kotropoulos, Constantine

Koukoulas, Triantafillos

Koulouriotis, Dimitrios E.

Kouretas, Dimitrios

Kouroussis, Georges

Kraft, Martin

Krajewski, Jarek

Kreimer, Joseph

Krishnaswami, Hariharan

Kromanis, Rolands

Kroschel, Kristian

Kruglov, Roman

Kshemkalyani, Ajay D.

Kuan, Chia-Yi

Kubiak, Tomasz

Kudo, Mineichi

Kulikov, Gennady

Kumar, Arunandan

Küng, Alain

Kunioka, Masao

Kuo, Chil-Chyuan

Kuperman, Alon

Kuremoto, Takashi

Kurumisawa, Kiyofumi

Kusch, Peter

Kwak, Hyo-Gyoung

Kwak, Moon K.

Kwan, Albert K. H.

Kwan, James

Kwapinska, Marzena

Kweon, Chol-Bum

Kwok, Ngai Ming

Kwon, Kwang-Chul

Kyuzi, Kamoi

La Mendola, Lidia

La Scalia, Giada

Lacava, Cosimo

Ladani, Leila

Lafoz, Marcos

Lagarde, Florence

Laheurte, Raynald
Lai, Ying-Chih

Lai, Yuan

Laity, Peter R

LaJeunesse, Dennis R.

Lam, Frank

Lam, Kwok-ho

Lamberti, Luciano

Lan, Chung Wen

Lanceros-Méndez, Senentxu

Landolfo, Raffaele

Lantieri, Claudio

Laory, Irwanda

Laplace, Patrick

Lapthorn, Adrian

Larsen, Peter Gorm

Laurell, Fredrik

Laurent, $\mathrm{Ch}$

Laurent, Jérôme

Laverty, David

Lavia, Lisa

Lavine, Adrienne

Lavrentieva, Antonina

Law, David W.

Lay-Ekuakille, Aimé

Leach, Richard

Lee, Bang Yeon

Lee, Cheng-Ming

Lee, Choon-Man

Lee, Christopher H. T.

Lee, Chul-Hee

Lee, Hak Jong

Lee, Heejo

Lee, Heon

Lee, Hyeong Jae

Lee, Hyoki

Lee, Hyoungsoon

Lee, Jeong Ik

Lee, Jong Jae

Lee, Joonhee

Lee, Ju-Yi

Lee, Ming-Tsang

Lee, Minjae

Lee, Moo-Yeon 
Lee, Sang Kwon

Lee, Suk Jin

Lee, Sun-Kyu

Lee, Wooyoung

Lee, Yih-Der

Lee, Yongchul

Lehmann, Peter

Lehner, Bernhard

Lehr, Claus-Michael

Leonesio, Marco

Leong, Jik Chang

Leou, Rong-Ceng

Lepidi, Marco

Leroux, Paul

Leva, Sonia

Levin, Dan

Lewinska, Anna

Li, Bingyun

Li, Dezhi

Li, Hao

Li, Huaizhong

Li, Jichao

Li, Kuo-Bin

Li, Long-yuan

Li, Mingda

Li, Qiaoxi

Li, Robert Kwok-Yiu

Li, Rui

Li, Silong

$\mathrm{Li}, \mathrm{Xi}$

Li, Yihan

Li, Yuwei

Li, Zili

Liang, C-T

Liang, Feng

Liang, Jinyang

Liang, Xihui

Liao, Jan-Ray

Liaw, Chang-Ming

Lih, Shu

Lim, Seung-Ho

Lin, Chih-Jer

Lin, Chin-Feng
Lin, Gong-Ru

Lin, Sheam-Chyun

Lin, Shinfeng D.

Lindborg, PerMagnus

Lindeberg, Tony

Lindström, Tom

Lineares, Jean-Marc

Ling, Steve

Lingham, Anthony R.

Linke, Heiner

Linn, Eike

Linß, Sebastian

Liou, Jing-chiou

Liou, Juin J

Liu, Bin

Liu, Cheng-Hsien

Liu, Cheuk Lun

Liu, Chunhua

Liu, Mengyang

Liu, Qing

Liu, Sung-Po

Liu, Tzong-shi

Liu, William

Liu, Yanjun

Liu, Ying-Ling

Liu, Yuanzhi

Lizcano, David

Lo Conte, Antonietta

Lo, Chun-Hao

Lo, Guo-Qiang

Lo, Men-Tzung

Loftness, Vivian

Lokki, Tapio

Longstaff, Andrew Peter

López De Lacalle Marcaide, Luis N.

Lopez, Gartzen

López, Miguel A.

López-Fernández, Carlos

Lopez-Linares, Francisco

López-López, José A.

López-Padilla, Alexis

Lopez-Rojas, Edgar Alonso

Lorenzo, Encarnación
Lorenzo, Miguel

Lostado, Ruben

Lotnyk, Andriy

$\mathrm{Lu}$, Junpeng

Lu, Meng

Lu, Ming

$\mathrm{Lu}$, Tao

Lu, Ting

Lu, Ya Yan

Luchetta, Antonio

Luigia, Sabbatini

Lundén, Janne

Luo, Zhiyuan

Luong, Dzung Dinh

Ly, Thuc Hue

Lyons, Pádraig

Lysenko, Dmitry A.

$\mathrm{Ma}, \mathrm{Ke}$

Ma, Wenxiu

Ma, Yue

Ma, Yuting

Mabwe, Augustin Mpanda

Maceli, Monica

Machado, Jose

Machimbarrena, María

Mackay, Ruth

Madimenos, Felicia

Maffei, Luigi

Maffucci, Antonio

Magliulo, Gennaro

Magno, Michele

Mahadeva, Suresha K.

Mahdi, Samira

Maicke, Brian

Maisch, Tim

Majima, Yutaka

Makarewicz, Oliwia

Makarov, Valeri A.

Mäkinen, Saku

Makino, Yoshio

Maksoud, Talal

Malekian, Reza

Malik, Farhan H. 
Mallardo, Vincenzo

Malureanu, Radu

Malvezzi, Monica

Mammone, Nadia

Mancini, Marco

Mander, John

Mangini, Agostino Marcello

Manhartsgruber, Bernhard

Manin, Julien

Manorathna, Prasad

Manos, George C.

Manske, Eberhard

Mansour, Gabriel

Manzello, Samuel L.

Mao, Chuanbin

Mao, Wei-Lung

Mao, Xiaoan

Maqsoud, Abdelkabir

Marchand, Sylvain

Marcolin, Federica

Marcos, Paula M.

Mariano, Sílvio José Pinto Simões

Marie, Emmanuelle

Marinakis, Vangelis

Marino, Joseph

Mark, Kedzierski

Markides, Christos

Markopoulos, Angelos P.

Marques, Rui

Martens, William L.

Martin Cordero, Carmen

Martín, Antonio E.

Martin, Jean-Michel

Martinelli, Enzo

Martinez Dominguez, Francisco

Martinez Krahmer, Daniel

Martinez-Hernandez, Uriel

Martins, Albino

Martins, João Poças

Martins, Pedro

Marty, Julien

Maruyama, Kazuo

Marzband, Mousa
Masoudian, Mohamad

Masrur, Alejandro

Mateos, Xavier

Mateos-Timoneda, Miguel A

Matos, J Nuno

Matsukawa, Mami

Matter, Michelle L.

Mavromatidis, Lazaros

Maxwell, Thomas M.R.

Mazeika, Dalius

Mazumdar, Sagnik

Mazza, Andrea

Mazza, Fabio

McAndrew, Anthony R.

Mcnelley, Terry R.

McPheron, Benjamin D

Medine, David

Mehdipour, Iman

Meher, Pramod Kumar

Mei, Yi

Meier, Samuel M.

Meister, Michael

Mekki, Othman Ben

Melo-Pinto, Pedro

Melskens, Jimmy

Memoli, Gianluca

Menendez Ramos, José Carlos

Meng, Lijian

Menon, Rani

Meola, Carosena

Mercado, Karla P.

Mercorelli, Paolo

Merola, Simona

Meroni, Daniela

Mertins, Alfred

Messner, Andreas

Metternicht, Graciela

Mezzavilla, Marco

Miao, Jiashi

Miccio, Francesco

Michailides, Constantine

Michailidis, George

Micheli, Davide
Mien, Van

Migallon, Hector

Millar, Graeme

Miller, David C.

Miller, Jimmie

Minakata, Hideaki

Mingareev, Ilya

Miranda, Cristobal

Miroshnichenk, Andrey E.

Mishra, Yogendra Kumar

Misra, R. Devesh K.

Missana, Tiziana

Mita, Damiano Gustavo

Miyaji, Hirofumi

Modarres, Mohammad

Modelli, Alberto

Moghadasi, Faridani Hadi

Mohan, Rajesh Elara

Molinari, Filippo

Momeni, Kasra

Momoniat, Ebrahim

Möncke, Doris

Monsurrò, Pietro

Monti, Alessio

Montufar, Guido F.

Montuori, Rosario

Moochhala, Shabbir M.

Moon, Jongsub

Moon, Jong-yun

Mora, Andre

Morabito, Francesco Carlo

Morales-Cid, Gabriel

Morelli, Francesco

Morelli, Michele

Moreno, Daniel

Moreno, Francisco-Angel

Morganti, Pierfrancesco

Moshou, Dimitrios

Mosk, Allard

Mossi, Karla

Motta, Gianmario

Mottola, Fabio

Mouchet, Sébastien 
Mounaix, Patrick

Moura, Pedro S.

Moysan, Joseph

Mrad, Nezih

Mucha, Jacek

Muiesan, Maria Lorenza

Muller, Andrew C.

Müller, Johannes

Müller, Meinard

Muñiz, Pilar

Munkelt, Christoph

Munoz, Macarena

Muñoz-Gea, Juan Pedro

Murray, John M.

Musleh, Basam

Muthuswamy, Karthik

Muttaqi, Kashem M.

Nadal Soriano, Enrique

Nagaoka, Kenji

Nagata, Fusaomi

Nagina, Parmar

Nagl, Stefan

Nairn, David G.

Najm, Husam

Nakamura, Hajime

Nakanishi, Shinsuke

Nakata, Toshiyuki

Nam, Doan Ngoc Chi

Nam, Jae-Do

Nam, Juhan

Nandi, Dipankar

Nanni, Loris

Naranjo, José Eugenio

Naser, Mohannad

Nasrollahi, Amir

Nath, Manashi

Naugler, Christopher

Navarro, Roberto

Navarro-Alarcon, David

Nazare, Shonali

Nejad, Amir R.

Nekhai, Sergei

Neto, Diogo Mariano
Neubauer, Reinhard

Neubert, Jeremiah

Ng, Selina S. Y.

Nganguia, Herve

Ngoko, Yanik

Nguyen, Hung

Nguyen, Kim-Doang

Nguyen, Nghia Q.

Nguyen, Tu Dinh

Nguyen, Vu Huy

$\mathrm{Ni}$, Bingbing

Nie, Mengyan

Nielsen, Heber

Niendorf, Thomas

Nikolaou, Michael

Nikolova, Dessislava

Nishio, Mayuko

Nobili, Andrea

Noboru, Yuasa

Nocera, Francesco

Nolte, Stefan

Nonino, Carlo

Nordahl, Rolf

Norman, Gethin

Norton, Michael

Noubactep, Chicgoua

Nour, Omer

Novotny, Lukas

Nozato, Hideaki

Ntontin, Konstantinos

Ntziachristos, Vasilis

Nunez, Alfredo

Oberst, Sebastian

Ochalski, Tomasz

Odeh, Suhail M.

Oenema, Oene

Ogata, Satoshi

Oh, Heekuck

$\mathrm{Oh}, \mathrm{Min}$

Oh, Teresa

Ohshima, Takashi

Ohtsu, Masayasu

Okayasu, Mitsuhiro
Oliveira, Hugo M.

Oliveira, João Pedro

Olivera, Pascual

Olyaee, Saeed

Ombres, Luciano

Omori, Toshihiro

Oneto, Luca

Ono, Kanji

Onogi, Shinya

Onoue, Kozo

Onoue, Kyohei

Ordoudi, Stella

Orlando, Maurizio

Orlik, Bernd

Orsini, Andrea

Ortelli, Simona

Ortiz, Alberto

Oswald, Jay

Ou, Ting-Chia

Ouagne, Pierre

Ouyang, Xiaoguang

Oza, Nikunj

Ozaki, Shingo

Ozbay, Kaan

Ozevin, Didem

Pachnicke, Stephan

Padmavathy, Kankanala

Paglialonga, Alessia

Pagnini, Gianni

Pagonis, Dimitrios Nikolaos

Paipetis, Alkis S.

Paiva, Rafael

Pal, Anamitra

Palazzolo, Alan

Palomares-Salas, José Carlos

Pan, Chi-Ling

Pan, Huang Hsing

Pan, Liang

Pan, Wencheng

Pang, Jong-hwei S.

Pantaleo, Antonio Marco

Papaelias, Mayorkinos

Papakonstantinou, Georgios 
Papakostas, Nikolaos

Papanikolaou, Seraphim

Papoutsidakis, Michail G.

Paredes, Ferran

Pareschi, Fabio

Park, Chan Eon

Park, Cheolwoong

Park, Jae-Hyeung

Park, Jang-Su

Park, Jun-Hong

Park, Namje

Park, Sang-gyu

Park, Sehyun

Park, YongKeun (Paul)

Parker, Julian

Parkinson, Simon

Parrales, Miguel A.

Parshad, Rana

Parveen, Iffat

Pasetto, Marco

Pasqualotto, Roberto

Pasquier, Philippe

Passacantando, Mauro

Passaglia, Elisa

Passian, Ali

Pastore, Roberto

Patel, Vipulkumar Ishvarbhai

Patil, Devendra

Patra, Jayanta Kumar

Patricia Ramos, Patricia

Patricio, Jorge

Patwardhan, Siddharth V

Pau, Giovanni

Paulino, Nuno

Paulo, Joel Vera Cruz Preto

Payá Vayá, Guillermo

Pazur, Richard J.

Peace, Angela

Peças Lopes, João A.

Pedersen, Henrik

Pelecanos, Loizos

Peltokangas, Mikko

Pelusi, Danilo
Peng, Qian

Peng, Szu-Hsien

Pepe, Antonio

Peragón, Juan

Pereira, Eduardo

Pereira, Elsa Maria Vaz

Pereira, Telmo

Pérez-Carrasco, José Antonio

Pérez-Gil, Jesús

Perhinschi, Mario

Perinelli, Diego Romano

Peris, Alfredo

Pescapè, Antonio

Peterman, Kara

Petit, Lionel

Pfeifle, Florian

Phan, Hop

Phung, Quoc Tri

Pichler, Bernhard

Piera, Miquel Àngel

Pignaton De Freitas, Edison

Pilard, Laurence

Pilloni, Virginia

Piluso, Vincenzo

Pimm, Andrew

Pinar Pérez, Jesús María

Pindado, Santiago

Pinho Lopes, Margarida F.

Pinkse, Pepijn W. H.

Pinna, Luigi

Pinoy, Luc

Piras, Andrea

Pisano, Giuditta

Piva, Terrence

Plante, Jean-Sébastien

Platero, Carlos A.

Plech, Anton

Pleissner, Daniel

Plochocka, Paulina

Pluchery, Olivier

Plummer, Andrew

Podila, Ramakrishna

Poksawat, Pakorn
Poltschak, Florian

Pommerenke, David

Pompili, Riccardo

Poncet, Sébastien

Ponsot, Emmanuel

Portmann, Edy

Poti, Luca

Potortì, Francesco

Poulymenopoulou, Mikaela

Pourbabak, Hajir

Powell, Kody M.

Prabha, Swayam

Prada, Francesco

Precup, Radu-Emil

Preinfalk, Jan B.

Prendergast, Luke J.

Pretsch, Thorsten

Prinz, Torsten

Pryss, Rüdiger

Psychalinos, Costas

Pucci, Andrea

Puga, Hélder

Pyattaev, Alexander

Pyun, Young-Sik

Qin, Zian

Quentin, Noirhomme

Quintana, David

Ra, Youngchul

Rabenstein, Rudolf

Raboso, Mariano

Radamson, Henry $\mathrm{H}$.

Radcliffe, P. J.

Radhakrishnan, Chandra

Rahier, Hubert

Rahmani, Ramin

Rahnejat, Homer

Rakotonirainy, Andry

Ramalli, Alessandro

Ramasamy, Savitha

Ramasamy, Subramanian

Rambold, Holger A.

Rämö, Jussi

Rana, Dipak 
Rastegarpanah, Alireza

Rath, Jagat J.

Razzaq, Mudassar

Rebaudengo, Maurizio

Rees, Andrew

Reggiani, Susanna

Régis, Guégan

Reis, Gustavo

Reisslein, Martin

Ren, Shuxin

Renaud, Nicolas

Renevey, Philippe

Renò, Filippo

Renzi, Cristina

Retsch, Markus

Reverberi, Andrea

Rezazad, Mostafa

Rhee, Shin Hyung

Rhi, Seok-Ho

Rhinehart, R. Russell

Riba, Jordi-Roger

Ribeiro, Jorge

Ricco, Pierre

Rice, Aaron N.

Richter, Uwe

Riela, Serena

Righini, Giancarlo C.

Rivadeneira, Paula

Riziotis, Christos

Rizzo, Santi Agatino

Robertson, William M.

Roddaro, Stefano

Ródenas, Juan

Rodger, James A.

Rodriguez, Brian

Rodriguez, Ignacio

Rodríguez, Juan M. Corchado

Rodríguez-Couto, Susana

Rodriguez-Hernandez, Miguel A.

Rodriguez-Ubinas, Edwin

Roedel, Christian

Rogers, Eric

Romaniello, Roberto
Romero-Cadaval, Enrique

Romoli, Laura

Ronan, William

Rong, Libin

Roper, Donald Keith

Rosendahl, Lasse Aistrup

Rossi, René M.

Rostami, Mohammad

Roth, Stephan V.

Rovetta, Stefano

Rowe, W. Brian

Ruano, António

Rubin, Stuart H.

Ruffin, Paul

Ruiz Raga, Sonia

Ruiz-Cárcel, Cristobal

Rumley, Sébastien

Runge, Antoine

Russell, James R.

Russo, Pietro

Rustighi, Emiliano

Rychtarikova, Monika

Ryll, Markus

Saadeh, Mohammad

Sadhu, Ayan

Sae-Bae, Napa

Safacas, Athanasios

Saghezchi, Firooz B.

Sah, Hong-Kee

Sahajpal, Ritvik

Şahin, Fatma Ceyhun

Sajjadian, Masoud

Salim, Hani

Salvador, Francisco J.

Salzano, Ernesto

Sánchez De La Flor, Francisco J.

Sanchez-Rodriguez, David

Sangiorgi, Cesare

Sanguinetti, Paola

Sanquer, Marc

Sansotera, Maurizio

Santander, Javier

Santos, Catarina
Santos, João Pedro

Santulli, Carlo

Sanz, José Antonio

Sappa, Giuseppe

Saraiva, Lucilia

Sarasini, Fabrizio

Saruhan, Bilge

Saslis-Lagoudakis, C. Haris

Sato, Hiroyuki

Saurín, Noelia

Savija, Branko

Scamoni, Fabio

Scaramuzza, Andrea E

Schagaev, Igor

Schaller, Jeffrey

Schartel, Bernhard

Schirru, Michele

Schmitz, Gerhard

Schneider, Josef

Schneider, Michael

Schneider, Raphael

Scholz, Markus

Schreiber, Laura

Schwartz, Stanley A.

Scozzafava, Andrea

Secchi, Simone

Secco, Emanuele

Sedigh, Sahra

Segura Garcia, Jaume

Seguy, Sébastien

Selbekk, Tormod

Selci, Stefano

Semeraro, Teodoro

Sen, Bhaskar

Sena, Enzo

Seo, Kisung

Sepasi, Saeed

Serôdio, Carlos

Serrao, Carlos

Sescu, Adrian

Seshadri, Mukund

Sessa, Salvatore

Seyed-Aghazadeh, Banafsheh 
Sfarra, Stefano

Shabalin, Andrey A.

Shafik, Rishad

Shah, Lawrence

Shahjamali, Mohammad Mehdi

Shaker, George

Shamir, Lior

Shanwen, Tao

Shao, Chenhui

Shao, Peng

Sharma, Nidhi

She, Yu

Sherburn, Jesse A.

Sheu, Jeng-Shin

Sheu, Jia-Shing

Shi, Junping

Shi, Shih-Chen

Shi, Xianming

Shieh, Jiann

Shih, Yin-Tzer

Shikida, Mitsuhiro

Shimada, Keita

Shimizu, Yuki

Shokrani, Alborz

Siddiqui, Javed

Sidhu, Harvinder

Sieczkarek, Peter

Siegel, David

Sillanpaa, Mika

Silva, Luís R.

Simon, Rowland

Simoni, Daniele

Simpson, Garth J.

Sindelar, Mark F.

Sing, Swee Leong

Singamaneni, Srinivasa Rao

Sinha, Yashwant

Sirvio, Juho Antti

Sivasubramanian, Aravind

Sladek, Jerzy

Sloan, Robin

Slonecker, Terrence

Smalley, Joseph S. T.
Smets, Ilse Y.

Smirnov, Alexander

Smith, Hal

Smith, Ian

Soares, João

Soares, Vasco N.G.J.

Söffker, Dirk

Sohn, Il

Solanki, Sarika

Solberg, Ole Vegard

Soler, Cyril

Solís, Javier

Somani, Abhishek

Somani, Mahesh

Song, Gwo-Shyh

Song, Ju Bin

Song, Linqi

Song, Tian Yi

Song, Weimin

Sontacchi, Alois

Sookhak, Mehdi

Sorrentino, Luigi

Soshi, Masakazu

Soulat, Damien

Souli, M'hamed

Sousa, Jorge

Spataru, Catalina

Spinello, Davide

Srinivas, Keerthi

Srinivasan, Sudharsanan

Stagkos, Dimitri

Stanescu, Michaela Dina

Stanica, Razvan

Stano, Pasquale

Stara, Riccardo

Stavenga, Doekele

Stavrakakis, George

Stavroulakis, Georgios E.

Stegmayr, Carina

Steidl, Gabriele

Steinmann, Wolf-Dieter

Stelson, Kim A.

Stephenson, Uwe M.
Stetter, Ralf

Stillfried, Georg

Stoevesandt, Bernhard

Stonaha, Paul J.

Stoychev, Georgi

Strain, Michael J.

Strauss, Alfred

Strauss, Sarah

Strozzi, Antonio

$\mathrm{Su}$, Wencong

Suárez, Fernando

Sucasas, Victor

Sudarsono, Anugrah Sabdono

Sugawa, Kosuke

Suh, Young Soo

Sukumaran, Vijay

Summers, Joshua D.

Sun, Ruoyu

Sunderland, Keith

Sung, Dan Keun

Sung, Tae Hyun

Suntio, Teuvo

Suominen, Hanna

Surdilovic, Dragoljub

Sutherland, John B.

Suwada, Tsuyoshi

Svendsen, Svend

Swindle, Andrew

Swolfs, Yentl

Syam, Wahyudin P.

Sydlik, Stefanie A.

Syed, Zahid

Szabo, Alexandre

Szulczewski, Gregory

Tadinada, Aditya

Taghia, Jalal

Tagliabue, Giulia

Tajmir-Riahi, Heidar-ali

Takahashi, Yasuo

Takamasu, Kiyoshi

Takasugi, Takayuki

Takeya, Kei

Taki, Hirofumi 


\section{Takizawa, Makoto}

Tamburrano, Paolo

Tan, Hua

Tan, Jing

Tan, Noel Peter Bengzon

Tanaka, Atsuhiro

Tanaka, Manabu

Tanaka, Tadashi

Tang, Chun

Tang, Hansong

Tang, Shanshan

Tang, Youhong

Tankam, Patrice

Tao, Bing

Tao, Kai

Taormina, Riccardo

Tarczynski, Andrzej

Tardón, Lorenzo J.

Tava, Aldo

Tenbohlen, Stefan

Teng, Mao-Hua

Teo, Tat Joo

Thalmann, Rudolf

Theilen-Willige, Barbara

Thérias, Sandrine

Thern, Marcus

Thomas, Kavitha P

Thomases, Becca

Thompson, John R.

Thomy, Vincent

Throckmorton, Chandra S.

Tia, Mang

Tian, Fangbao

Tian, Gui Yun

Tian, Yuan

Tiraferri, Alberto

Tjahjowidodo, Tegoeh

Tollner, Ernest W.

Tomar, Vikrant Singh

Tombelli, Sara

Tomoyoshi, Nishiumura

Torija, Antonio J.

Tornabene, Francesco
Torres, Cristiana

Torres, David

Tosello, Guido

Touhy, Paul

Toupance, Thierry

Town, Graham

Tran, Gia Khanh

Traversi, Gianluca

Travieso-Gonzalez, Carlos M.

Trebino, Rick

Triantafillou, Thanasis

Trigueiros, Paulo

Triñanes, Pablo García

Troiani, Enrico

Tronchin, Lamberto

Trung, Ngo Thanh

Tsai, Miching

Tsai, Philbert

Tsao, Yu

Tsavdaridis, Konstantinos Daniel

Tsikalakis, Antonis G.

Tsow, Francis (Tsing)

Tsuji, Yasuhide

Tsuji, Yutaka

Tsuta, Mizuki

Tuluri, Francis

Tung, Steve

Turitsyn, Konstantin

Tursini, Marco

Turunen, Mikael J.

Twu, Ruey-Ching

Tzanetakis, George

Tzeng, Sheng-Chung

Tzou, Dayu

Ubertini, Filippo

Ueno, Takafumi

Uezu, Kazuya

Uhlmann, Eckart

Uhrlandt, Dirk

Upare, Pravin P.

Urban, Matthew W.

Vahedi, Hani

Vaiana, Rosolino
Välimäki, Vesa

Valles, Elisa

Valova, Iren

Valverde, Jose C.

Van Den Bergh, Hubert

Van Den Bossche, Nathan

Van Der Bruggen, Bart

Van Der Meer, Theo H.

Van Der Neut, Joost

Van Der Zwaag, Sybrand

Van Gemert, Jan

Van Haute, Sam

Van Hoof, Joost

Van Hulle, Marc

Van Humbeeck, Jan

Varela, Alejandro Cerda

Vargemezis, George

Vashist, Sandeep Kumar

Vasilakos, Athanasios

Vassilacopoulos, Georgios

Vassiliadis, Savvas

Vastola, Guglielmo

Vega, Gladys Mínguez

Vega-Rodríguez, Miguel A.

Veinot, Jonathan

Velazquez, Ramiro

Velimirovic, Milica

Vento, Mario

Venus, Joachim

Verma, Mohit S.

Vermeir, Gerrit

Victoria, Marta

Vidoni, Renato

Vien, Quoc-Tuan

Vignali, Valeria

Vilanova, Ramon

Viñas, Miguel

Vinding, Mads S.

Vinuesa, Ricardo

Virtanen, Tuomas

Visaveliya, Nikunjkumar

Visentin, Chiara

Visintin, Phillip 
Visser, Wilfried P. J.

Vitelli, Massimo

Viviano, Antonino

Vlachos, Nicholas

Vogel, Thomas

Voliani, Valerio

Vollgger, Stefan

Vollmer, Doris

Volos, Christos

Vorobieff, Peter

Vourkas, Ioannis

Vouyiouka, Stamatina N.

Vox, Giuliano

Vozel, Benoit

Wabnitz, Stefan

Wada, Yuji

Wagner, Bernardo

Wagner, Steven

Wagner, Thorsten

Wahid, Khan A.

Wang, Alan X.

Wang, Ching-Lin

Wang, De-Yi

Wang, Dong

Wang, Gai-Ge

Wang, Hai

Wang, Haining

Wang, Hsien-Chang

Wang, Jung-Chang

Wang, Junwei

Wang, Lei

Wang, Lipo

Wang, Ming-Shyan

Wang, Rongping

Wang, Xi Vincent

Wang, Xingwei

Wang, Yao-tien

Wang, Yukai

Wang, Zheng

Wang, Zuankai

Watanabe, Masanori

Watson, Des

Webber, Michael
Wei, Peng-Sheng

Wei, Xiong

Wei, Xiupeng

Welch, David

Welemane, Hélène

Wendzel, Steffen

Wensing, Patrick

Werner, Kurt

Wexler, Adam D.

Whelan, Matthew J.

Whiteley, Hugh

Wilhelm-Mauch, Frank

Willberg, Christian

Williams, Alan

Williams, Paul J

Williams, Wesley

Winzenrieth, Renaud

Wits, Wessel W.

Wojkiewicz, Jean-Luc

Wolak, Mason

Wollenhaupt, Matthias

Wong, Kok

Woo, Eamor M

Woodhouse, Jim

Woodward, R. I.

Wotawa, Franz

Woungang, Isaac

$\mathrm{Wu}$, Jianguo

Wu, Kevin

$\mathrm{Wu}$, Lei

$\mathrm{Wu}$, Tung-Kung

Wu, Xing

Wuest, Thorsten

Wünsche, Michael

Wurstbauer, Ursula

Wycisk, Ryszard

Xhonneux, André

Xiao, Boqi

Xiao, Yanyu

Xu, Weixing

Yakovlev, Vladislav S.

Yakovlev, Vladislav V.

Yamamoto, Masashi
Yan, Xing

Yan, Xinggang

Yanagida, Sayaka

Yang, Fang

Yang, Liangcheng

Yang, Mijia

Yang, Shiming

Yang, Steve Y.

Yang, Xin-She

Yang, Yang

Yang, Yeon-Mo

Yang, Yu-Chiao

Yao, Jian

Yao, Wu-Sung

Yastrebov, Vladislav A.

Yasuda, Muneki

Yazdani Nezhad, Hamed

Ye, Huaiyu

Yee, Ki-Ju

Yeh, Hongjin

Yeh, Kuo-Hui

Yen, Chih-Ta

Yen, Feng-Lin

Yen, Jia-Yush

Yeo, Swee Hock

Yeoh, Guan Heng

Yeon, Jung Heum

Yi, Jin-Hak

Yilmaz, Emre

Yim, Sehyuk

Yin, Hsin-Bai

Yin, Peng-Yeng

Ying, Kuo-Ching

Yoh, Shikoh

Yoon, Jungwon

Yoon, Sung-Guk

Yoshimoto, Shigeka

Yoshimura, Takeshi

Yoshitake, Isamu

Yost, Michael J.

You, Shingchern

Young, Wen-Bin

Yu, Qing Liang 


\begin{tabular}{l} 
Yu, Tzyy-Lung Leon \\
Yu, Xiangzhi \\
Yu, Xiao-Hua \\
Yu, Xin \\
Yu, Xun \\
Yu, Yang \\
Yu, Yi-Hsiang \\
Yu, Ziniu \\
Yue, Yang \\
Zabini, Flavio \\
Zabulis, Xenophon \\
Zacharof, Myrto-Panagiota \\
Zamani, Nematollah \\
Zambon, Giovanni \\
Zamboni, Giorgio \\
Zandi, Omid \\
Zanin, Massimiliano \\
Zarrelli, Mauro \\
Zarrouk, David \\
Zavadskas, Edmundas K. \\
Zeng, Jianwu \\
Zenger, Kai \\
\hline
\end{tabular}

Zervaki, Anna D.

Zhan, Haifei

Zhang, Chunwei

Zhang, Dahai

Zhang, Dan

Zhang, Haijun

Zhang, Jie

Zhang, Le

Zhang, Longhe

Zhang, Peng

Zhang, Tonghua

Zhang, Wei

Zhang, Weiguang

Zhao, Jiyun

Zhao, Yuming

Zheng, Fuchun

Zheng, Rencheng

Zheng, Yuanjin

Zhou, Jiani

Zhou, Jie

Zhou, Xiao Dong

Zhou, Xiaobo
Zhou, Xingguo

Zhou, Yuan

Zhou, Yufeng

Zhu, Boyuan

Zhu, Jianjun

Zhu, Mingming

Zhu, Peifen

Zhu, Zhe

Zhuge, Qunbi

Ziemann, Amanda

Zimmerman, William

Zin, Valentina

Zio, Enrico

Zona, Alessandro

Zorba, Nizar

Zou, Yi

Zubieta, Luis

Zuffanelli, Simone

Zuo, Jian

Zuzuarregui, Ana

Zygielbaum, Arthur

(C) 2017 by the authors; licensee MDPI, Basel, Switzerland. This article is an open access article distributed under the terms and conditions of the Creative Commons Attribution (CC-BY) license (http://creativecommons.org/licenses/by/4.0/). 